\title{
Hybrid protocol for wireless EH network over weibull fading channel: performance analysis
}

\author{
Phu Tran Tin ${ }^{1}$, Tan N. Nguyen ${ }^{2}$, Tran Thanh Trang ${ }^{3}$ \\ ${ }^{1}$ Faculty of Electronics Technology, Industrial University of Ho Chi Minh City, Vietnam \\ ${ }^{2}$ Wireless Communications Research Group, Faculty of Electrical and Electronics Engineering, \\ Ton Duc Thang University, Vietnam \\ ${ }^{3}$ National Key Laboratory of Digital Control and System Engineering, Vietnam
}

\begin{tabular}{l}
\hline \hline Article Info \\
\hline Article history: \\
Received Apr 20, 2019 \\
Revised Oct 4, 2019 \\
Accepted Oct 15, 2019 \\
\hline
\end{tabular}

Keywords:

Energy harvesting

Monte Carlo simulations

Outage probability

TSR-PSR

\begin{abstract}
In this paper, the hybrid TSR-PSR protocol for wireless energy harvesting $(\mathrm{EH})$ relaying network over the Weibull fading channel is investigated. The system network is working in half-duplex (HD) mode. For evaluating the system performance, the closed-form and integral-form expressions of the outage probability (OP) are investigated and derived. After that, numerical results convinced that our derived analytical results are the same with the simulation results by using Monte Carlo simulation. This paper provides a novel recommendation for the wireless EH relaying network.
\end{abstract}

\section{Corresponding Author:}

Tan N. Nguyen,

Wireless Communications Research Group,

Faculty of Electrical and Electronics Engineering,

Ton Duc Thang University, Ho Chi Minh City, Vietnam.

Email: nguyennhattan@tdtu.edu.vn

\section{INTRODUCTION}

Due to the rapid development of the Internet of Things (IoT), 5G needs to support the massive connectivity of users and/or devices to meet the demand for low latency, low-cost devices, and diverse service types. However, energy harvesting (EH) and information transmission (IT) processes from the source (S) node to the destination (D) node in the communication network may be interrupted by fading, shadowing, and path loss. For solving this problem, the intermediate helping relay (R) node between the S node and D node is considered as an excellent solution for enhancing the system performance [1-4]. There are many types of research focused on the WCPN with helping the intermediate relay with perfect and imperfect channel state information (CSI) as investigated in [5-7]. The authors in [8] investigated the fault-tolerant schemes with the presence of imperfect CSI. Two primary protocols Half-duplex (HD) and Full-duplex (FD) are popularly used in the relaying communication network. HD mode based on the simple design and implementation is popularly used in the traditional wireless power communication networks. But the HD scheme can transmit and receive the signal at the same time in the same frequency band, and then the HD scheme has a massive spectrum efficiency loss [9-11]. In comparison with the HD scheme, the FD scheme can receive and transmit signals continuously and can achieve up to double the capacity. In addition, the FD can reduce spectral loss with the innovative in the antenna technology [12-14]. Based on these problems, the hybrid TSR-PSR protocol can be considered as a novel solution for improving the system performance of the wireless EH relaying network. 
In this paper, we propose and investigate the hybrid TSR-PSR protocol for wireless energy harvesting $(\mathrm{EH})$ relaying network over the Weibull fading channel in half-duplex (HD) mode. To evaluate the performance of the proposed system, the closed-form and integral-form expression of the outage probability (OP) is analyzed and derived. Numerical results confirm that our obtained analytical results match well with the Monte Carlo simulations in connection with all possible system parameters. This paper provides a novel recommendation for the wireless $\mathrm{EH}$ relaying network. The main contributions of this research can be focused on as the followings:

a. We propose and investigate the hybrid TSR-PSR protocol for wireless EH relaying network over the Weibull fading channel in HD mode.

b. We derive the closed-form and integral-form expressions of the OP.

c. The influence of all main system parameters on the outage probability is investigated and discussed.

d. All results are verified by the Monte Carlo simulation.

\section{SYSTEM MODEL AND SYSTEM PERFORMANCE ANALYSIS}

The EH relaying network is illustrated in Figure 1. The energy harvesting (EH) and information transmission (IT) of the model system are presented in Figure 2. In Figure 2, we denote $\mathrm{T}$ is the block time of the EH and IT processes [13-15].

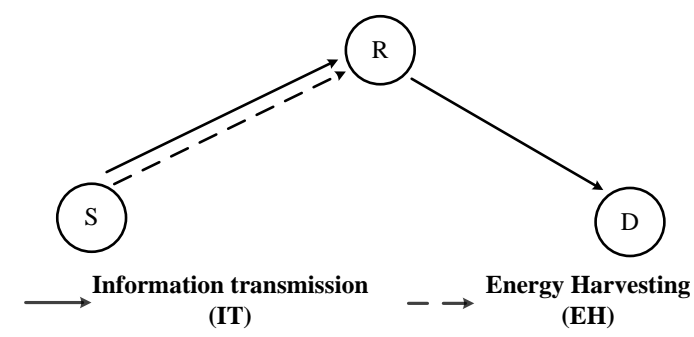

Figure 1. System model

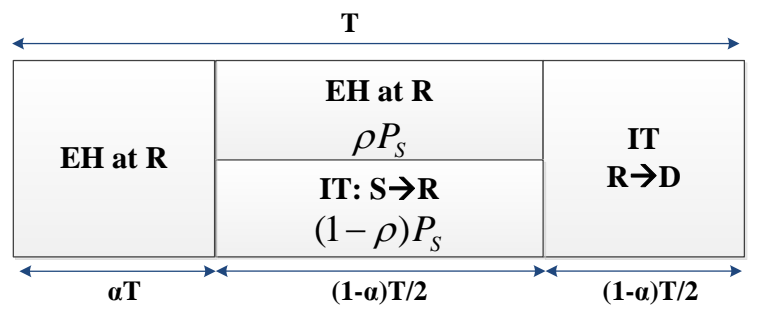

Figure 2. EH and IT processes

\subsection{Energy harvesting phase}

The received signal at the relay $\mathrm{R}$ in the first interval time slot $\alpha T$ can be formulated as

$$
y_{r}^{1}=\sqrt{P_{s}} h x_{s}+n_{r}
$$

Where $P_{s}$ is the transmitting power of source $S$, $h$ is channel gain and $n_{r}$ denoted the additive white Gaussian noise (AWGN) which has zero-mean and variance $\mathrm{N}_{0}$

$$
\mathrm{E}\left\{\left|x_{s}\right|^{2}\right\}=1 \text { which } \mathrm{E}\{\bullet\} \text { is the expectation operator and } x_{s} \text { is the energy symbol. }
$$

Based on (1), the harvested energy in the first timeslot can be given by

$$
E_{1}=\eta \alpha T P_{s}|h|^{2}
$$

Where $0<\eta \leq 1$ is the energy conversion efficiency. 
The received signal at $\mathrm{R}$ in the second time slot can be expressed as

$$
y_{r}^{2}=\sqrt{\rho P_{s}} h x_{s}+n_{r}
$$

Therefore, the harvested energy in this time slot also can be given by

$$
E_{2}=\eta \rho\left(\frac{1-\alpha}{2}\right) T P_{s}|h|^{2}
$$

From (2) and (4), the total average transmitted power at the relay $\mathrm{R}$ can be obtained as

$$
P_{r}=\frac{E_{r}}{(1-\alpha) T / 2}=\frac{E_{1}+E_{2}}{(1-\alpha) T / 2}=\kappa \eta P_{s}|h|^{2}
$$

Where $\kappa=\frac{2 \alpha+(1-\alpha) \rho}{1-\alpha}$

\subsection{Information transmission phase}

In the second interval time slot, the received signal at the relay $\mathrm{R}$ can be expressed as

$$
y_{r}=\sqrt{(1-\rho) P_{s}} h x_{s}+n_{r}
$$

In this model, we will consider the Amplify and Forward (AF) protocol. Hence the received signal and transmitted signal at the relay $\mathrm{R}$ have a relationship with the following which denoted amplifying factor

$$
\chi=\frac{x_{r}}{y_{r}}=\frac{1}{\sqrt{(1-\rho) P_{s}|h|^{2}+N_{0}}}
$$

Where $x_{r}$ is the transmitting signal at $\mathrm{R}$

In the third time slot, the received signal at the destination $\mathrm{D}$ can be given as

$$
y_{d}=g x_{r}+n_{d}
$$

Where $g$ is channel gain of R-D link and $n_{d}$ is AWGN at the destination which has zero-mean and variance $\mathrm{N}_{0}$ Substituting (6), (7) into (8), we have:

$$
\begin{aligned}
y_{d} & =g x_{r}+n_{d}=g \chi y_{r}+n_{d}=g \chi\left\{\sqrt{(1-\rho) P_{s}} h x_{s}+n_{r}\right\}+n_{d} \\
& =\underbrace{g \chi \sqrt{(1-\rho) P_{s}} h x_{s}}_{\text {signal }}+\underbrace{g \chi n_{r}+n_{d}}_{\text {noise }}
\end{aligned}
$$

The overall signal to noise ratio (SNR) from S to D can be obtained from (9)

$$
\gamma_{S R D}=\frac{\mathrm{E}\left\{\mid \text { signal }\left.\right|^{2}\right\}}{\mathrm{E}\left\{\mid \text { noise }\left.\right|^{2}\right\}}=\frac{(1-\rho) P_{s}|h|^{2}|g|^{2} \chi^{2}}{|g|^{2} \chi^{2} N_{0}+N_{0}} \approx \frac{\kappa \eta(1-\rho) \Delta X Y}{\kappa \eta Y+(1-\rho)}
$$

Where $X=|h|^{2}, Y=|g|^{2}, \Delta=\frac{P_{s}}{N_{0}}$

\section{Remark}

The cumulative density function (CDF) and probability density function (PDF) of random variable (RV) X, Y can be calculated by [14], respectively. 


$$
F_{i}(x)=1-\exp \left[-\left(\frac{x}{a}\right)^{b}\right]
$$

Where $i \in(X, Y)$, a and b are the Weibull parameter. From (11), PDF of X, Y can be obtained as

$$
f_{i}(x)=\frac{b}{a}\left(\frac{x}{a}\right)^{b-1} \exp \left[-\left(\frac{x}{a}\right)^{b}\right]
$$

\subsection{Outage probability (OP) analysis}

The OP of the system can be defined as

$$
O P=\operatorname{Pr}\left(\gamma_{S R D}<\gamma_{t h}\right)
$$

Where $\gamma_{t h}=2^{2 R}-1$ is the threshold of system and R: source rate

Substituting (10) into (13), we have

$$
\begin{aligned}
O P & =\operatorname{Pr}\left(\frac{\kappa \eta(1-\rho) \Delta X Y}{\kappa \eta Y+(1-\rho)}<\gamma_{t h}\right)=\operatorname{Pr}\left[X<\frac{\gamma_{t h}}{(1-\rho) \Delta}+\frac{\gamma_{t h}}{\kappa \eta \Delta Y}\right] \\
& =\int_{0}^{\infty} F_{X}\left[\frac{\gamma_{t h}}{(1-\rho) \Delta}+\frac{\gamma_{t h}}{\kappa \eta \Delta Y} \mid Y=y\right] f_{Y}(y) d y
\end{aligned}
$$

Combine with (11), (12), (14) can be rewritten as

$$
O P=1-\frac{b}{a^{b}} \int_{0}^{\infty} y^{b-1} \times \exp \left[-\frac{1}{a^{b}}\left(\frac{\gamma_{t h}}{(1-\rho) \Delta}+\frac{\gamma_{t h}}{\kappa \eta \Delta y}\right)^{b}\right] \times \exp \left[-\left(\frac{y}{a}\right)^{b}\right] d x
$$

Special case

When $b=1$ Weibull fading will correspond to the Rayleigh fading channel

The (15) can be reformulated as

$$
O P=1-\frac{1}{a} \exp \left[-\frac{\gamma_{t h}}{a(1-\rho) \Delta}\right] \int_{0}^{\infty} \exp \left(-\frac{\gamma_{t h}}{\kappa \eta \Delta y}\right) \exp \left(-\frac{y}{a}\right) d x
$$

Apply eq [3.324,1] of table of integral [16], The (16) can be rewritten as

$$
O P=1-2 \exp \left[-\frac{\gamma_{t h}}{a(1-\rho) \Delta}\right] \times \sqrt{\frac{\gamma_{t h}}{a \kappa \eta \Delta}} K_{1}\left(2 \sqrt{\frac{\gamma_{t h}}{a \kappa \eta \Delta}}\right)
$$

Where $K_{v}(\bullet)$ is the modified Bessel function of the second kind and $\mathrm{v}^{\text {th }}$ order.

\section{RESULTS AND AND DISCUSSION}

For validation, the correctness of the derived system performance expressions, as well as investigation of the effect of various parameters on the system performance, a set of Monte Carlo simulations are conducted in this section [17-25]. The system OP versus $\alpha$ and $\rho$ is plotted in Figure 3 with basic system parameters as $b=0.5, \Delta=10 \mathrm{~dB}$. In this analysis, the $\alpha$ and $\rho$ vary from 0 to 1 continuously. From the research results, the system OP increases significantly with rising the $\alpha$ and $\rho$ to 1 . All the simulation and analytical curves matched well with each other.

Furthermore, Figure 4 shows the connection between the system OP and TS factor $\alpha$ with $\mathrm{R}=0.5$ bps, $\rho=0.5$ and $\eta=0.8$. As shown in Figure 4 , we can see that the system OP has a decrease when the TS factor $\alpha$ varies from 0 to 1 in connection with the fact that more power is used for harvesting energy at $\mathrm{R}$ than 
power is used for information transmission between D,R and S. Again all simulation and analytical results agree well with each other.

Moreover, the function of the OP on the energy conversion efficiency $\eta$ is illustrated in Figure 5. Here we set the main system parameters as $\mathrm{R}=0.5 \mathrm{bps}, \Delta=5 \mathrm{~dB}$, and $\mathrm{b}=0.5$. Similar to the above cases, the $\mathrm{OP}$ of the model system increase crucially while the energy conversion efficiency varies from 0 to 1 . It can be observed that the more efficient energy conversion of the system the less outage probability. In addition, the analytical curve is the same as the simulation curve as shown in Figure 5. Finally, the OP versus the source rate $\mathrm{R}$ is shown in Figure 6 with $\alpha=0.5, \eta=0.8$ and $\gamma_{0}=5,10 \mathrm{~dB}$. From the results, the OP increases significantly when the source rate increases from 0 to 7 . We can see that the simulation and the analytical result are the same with all values of the source rate $\mathrm{R}$.

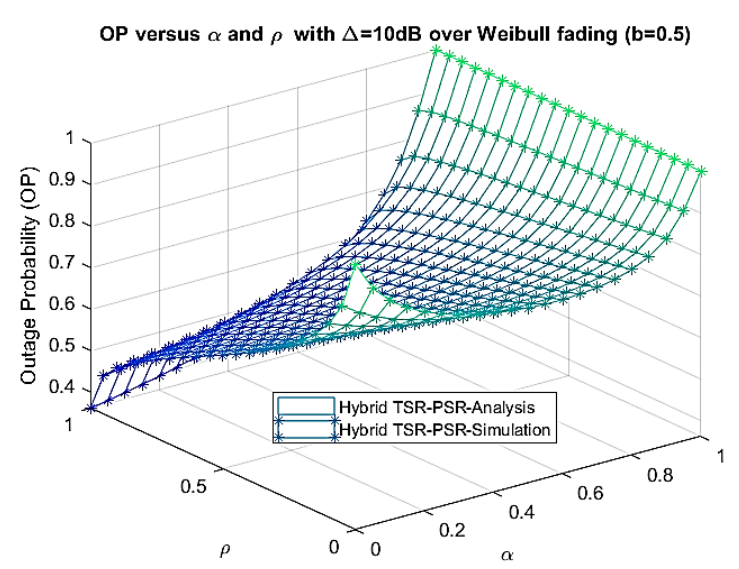

Figure 3. OP versus $\alpha$ and $\rho$

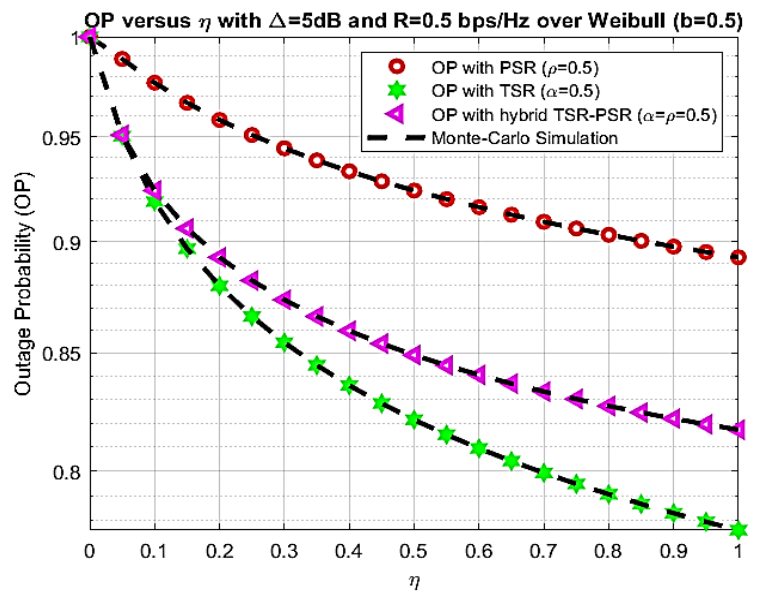

Figure 5. OP versus $\eta$

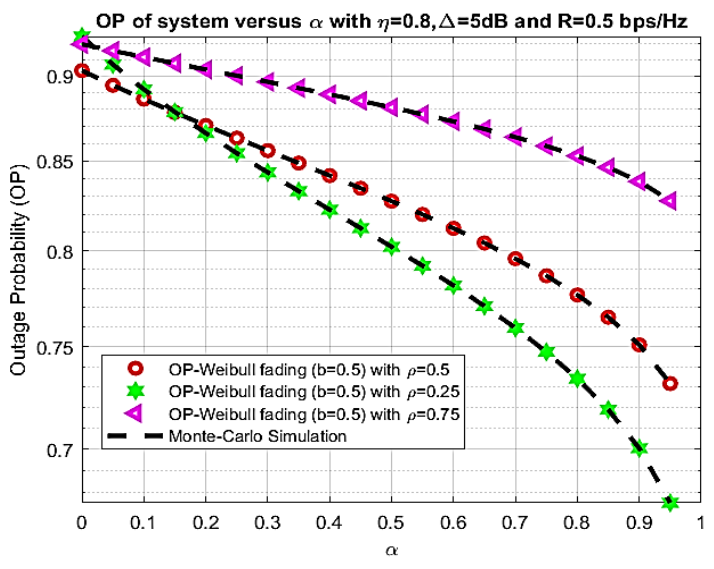

Figure 4. OP versus $\alpha$

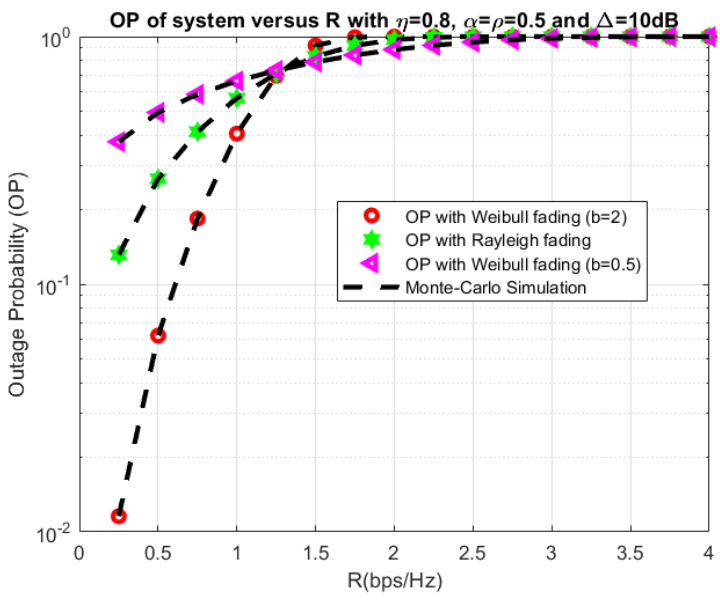

Figure 6. OP versus R

\section{CONCLUSION}

In this paper, the hybrid TSR-PSR protocol for wireless EH relaying network over the Weibull fading channel in HD mode is investigated. To evaluate the performance of the proposed system, the closedform and integral-form expression of the OP is analyzed and derived. Numerical results confirm that our derived analytical results match well with the Monte Carlo simulations in connection with all possible system parameters. This paper provides a recommendation for the wireless $\mathrm{EH}$ relaying network

\section{ACKNOWLEDGEMENTS}

This research was supported by National Key Laboratory of Digital Control and System Engineering (DCSELAB), HCMUT, VNU-HCM, Vietnam. 


\section{REFERENCES}

[1] Chen, He, Chao Zhai, Yonghui Li, and Branka Vucetic, "Cooperative Strategies for Wireless-Powered Communications: An Overview," IEEE Wireless Communications, vol. 25, no. 4, pp. 112-19, 2018. doi:10.1109/mwc.2017.1700245.

[2] Yu, H., Lee, H., and Jeon, H., "What is 5G? Emerging 5G Mobile Services and Network Requirements," Sustainability, vol. 9, pp. 1848, 2017.

[3] Sharma, V., and Karmakar, P.,"A Novel Method of Opportunistic Wireless Energy Harvesting in Cognitive Radio Networks," 2015 7thInternational Conference on Computational Intelligence, CommunicationSystems and Networks, 2015. doi:10.1109/cicsyn.2015.21.

[4] Boccardi, Federico, Robert Heath, Angel Lozano, Thomas Marzetta, and Petar Popovski, "Five Disruptive Technology Directions for 5G," IEEE Communications Magazine, vol. 52, no. 2, pp. 74-80, 2014. doi:10.1109/mcom.2014.6736746.

[5] Nasir, Ali A., Xiangyun Zhou, Salman Durrani, and Rodney A. Kennedy, "Relaying Protocols for Wireless Energy Harvesting and Information Processing," IEEE Transactions on Wireless Communications, vol. 12, no. 7, pp. 3622-3636, 2013. doi:10.1109/twc.2013.062413.122042

[6] Choi, Dongwook, and Jae Hong Lee, "Outage Probability of Two-Way Full-Duplex Relaying With Imperfect Channel State Information," IEEE Communications Letters, vol. 18, no. 6, pp. 933-936, 2014. doi:10.1109/lcomm.2014.2320940.

[7] Z. Ding, C. Zhong, D. W. K. Ng, M. Peng, H. A. Suraweera, R.Schober and H. V. Poor, "Application of smart antenna technologies in simultaneous wireless information and power transfer," IEEE Commun. Mag., vol. 53, pp. 86-93, Apr. 2015.

[8] Tourki, Kamel, Khalid A. Qaraqe, and Mohamed-Slim Alouini, "Outage Analysis for Underlay Cognitive Networks Using Incremental Regenerative Relaying," IEEE Transactions on Vehicular Technology, vol. 62, no. 2, pp. 721-734, 2013. doi:10.1109/tvt.2012.2222947.

[9] Okandeji, Alexander A., Muhammad R. A. Khandaker, and Kai-Kit Wong, "Wireless Information and Power Transfer in Full-Duplex Communication Systems," 2016 IEEE International Conference on Communications (ICC), 2016. doi:10.1109/icc.2016.7511169.

[10] Nasir, Ali A., Xiangyun Zhou, Salman Durrani, and Rodney A. Kennedy, "Throughput and Ergodic Capacity of Wireless Energy Harvesting Based DF Relaying Network," 2014 IEEE International Conference on Communications (ICC), 2014. doi:10.1109/icc.2014.6883957.

[11] Baidas, Mohammed W., and Emad A. Alsusa, "Power Allocation, Relay Selection and Energy Cooperation Strategies in Energy Harvesting Cooperative Wireless Networks," Wireless Communications and Mobile Computing, vol. 16, no. 14, pp. 2065-2082, 2016. doi:10.1002/wcm.2668.

[12] H. A. Suraweera, I. Krikidis, G. Zheng, C.Yuen and P. J. Smith, "Low-complexity end-to-end performance optimization in MIMO full-duplex relay systems," IEEE Trans. Wireless Commun., vol. 13, pp. 913-927, Feb. 2014.

[13] C. Zhong, H. A. Suraweera, G. Zheng, I. Krikidis and Z. Zhang, "Wireless Information and Power Transfer With Full-Duplex Relaying," in IEEE Transactions on Communications, vol. 62, no. 10, pp. 3447-3461, Oct. 2014. doi: 10.1109/TCOMM.2014.2357423

[14] A. Chaminda, J. Samarasekera, Dac-Binh Ha, H. K. Nguyen, "Best relay selection for underlay cognitive relaying networks over Weibull fading channels," Proc. of ComManTel 2014, 2014.

[15] Nasir, Ali A., Xiangyun Zhou, Salman Durrani, and Rodney A. Kennedy, "Relaying Protocols for Wireless Energy Harvesting and Information Processing," IEEE Transactions on Wireless Communications, vol. 12, no. 7, pp. 3622-636, 2013. doi:10.1109/twc.2013.062413.122042.

[16] Table of Integrals, Series, and Products, 2015. doi:10.1016/c2010-0-64839-5.

[17] Zhong, Caijun, Shi Jin, Kai-Kit Wong, and Matthew R. Mckay, "Ergodic Mutual Information Analysis for MultiKeyhole MIMO Channels," IEEE Transactions on Wireless Communications, vol. 10, no. 6, pp. 1754-763, 2011. doi:10.1109/twc.2011.041311.091011.

[18] Nguyen, Tan N., Minh Tran, Phuong T. Tran, Phu Tran Tin, Thanh-Long Nguyen, Duy-Hung Ha, and Miroslav Voznak, "On the Performance of Power Splitting Energy Harvested Wireless Full-Duplex Relaying Network with Imperfect CSI over Dissimilar Channels," Security and Communication Networks 2018, pp. 1-11, 2018. doi:10.1155/2018/6036087.

[19] Nguyen, Tan N., Tran Hoang Quang Minh, Phuong T. Tran, Miroslav Voznak, Tran Trung Duy, Thanh-Long Nguyen, and Phu Tran Tin, "Performance Enhancement for Energy Harvesting Based Two-way Relay Protocols in Wireless Ad-hoc Networks with Partial and Full Relay Selection Methods," Ad Hoc Networks, vol. 84, pp. 178-87, 2019. doi:10.1016/j.adhoc.2018.10.005.

[20] Nguyen, Tan, Phu Tran Tin, Duy Ha, Miroslav Voznak, Phuong Tran, Minh Tran, and Thanh-Long Nguyen, "Hybrid TSR-PSR Alternate Energy Harvesting Relay Network over Rician Fading Channels: Outage Probability and SER Analysis," Sensors, vol. 18, no. 11, pp. 3839, 2018. doi:10.3390/s18113839.

[21] Nguyen, Tan, Tran Quang Minh, Phuong Tran, and Miroslav Vozńák, "Energy Harvesting over Rician Fading Channel: A Performance Analysis for Half-Duplex Bidirectional Sensor Networks under Hardware Impairments," Sensors, vol. 18, no. 6, pp. 1781, 2018. doi:10.3390/s18061781.

[22] Nguyen, Tan N., Phuong T. Tran, Tran Hoang Quang Minh, Miroslav Voznak, and Lukas Sevcik, "Two-Way Half Duplex Decode and Forward Relaying Network with Hardware Impairment over Rician Fading Channel: System Performance Analysis," Elektronika Ir Elektrotechnika, vol. 24, no. 2, 2018. doi:10.5755/j01.eie.24.2.20639. 
[23] Tin, Phu Tran, Minh Tran, Tan N. Nguyen, and Thanh-Long Nguyen, "A New Look at Energy Harvesting Halfduplex DF Power Splitting Protocol Relay Network over Rician Channel in Case of Maximizing Capacity." Indonesian Journal of Electrical Engineering and Computer Science (IJEECS), vol. 13, no. 1, pp. 249, 2019. doi:10.11591/ijeecs.v13.i1.pp249-257.

[24] Tin, Phu Tran, Le Anh Vu, Tan N. Nguyen, and Thanh-Long Nguyen. "User Selection Protocol in DF Cooperative Networks with Hybrid TSR-PSR Protocol Based Full-duplex Energy Harvesting over Rayleigh Fading Channel: System Performance Analysis." Indonesian Journal of Electrical Engineering and Computer Science (IJEECS), vol. 13, no. 2, pp. 534, 2019. doi:10.11591/ijeecs.v13.i2.pp534-542.

[25] Tin, PhuTran, Minh Tran, Tan N. Nguyen, and Thanh-Long Nguyen, "System Performance Analysis of Hybrid Time-power Switching Protocol of EH Bidirectional Relaying Network in Amplify-and-forward Mode," Indonesian Journal of Electrical Engineering and Computer Science (IJEECS), vol. 14, no. 1, vol. 118, 2019. doi:10.11591/ijeecs.v14.i1.pp118-126. 